\title{
Recent Developments in Multiple-Scattering Calculations of XAFS and XANES
}

\author{
J.J. REHR \\ Dept. of Physics, FM-15, University of Washington, \\ Seattle, WA 98195 USA
}

\begin{abstract}
Curved-wave multiple-scattering (MS) theory provides a unified treatment of XAFS, encompassing both EXAFS and XANES, as well as a formal equivalence between exact treatments and the (MS) expansion carried to all orders. Recent developments in the theory of XAFS are reviewed with an emphasis on progress in curved-wave MS calculations and on understanding the nature of MS contributions in EXAFS and XANES. In particular we discuss a unified $a b$ initio MS treatment of XAFS based on a scattering matrix formalism which has sufficient speed and accuracy to treat high-order MS in extended systems. We find that neither low-order MS nor full MS theories are fully satisfactory. Instead, sufficiently high-order MS appears to be necessary for the convergence of both EXAFS and XANES calculations. Our approach also gives a MS interpretation of the $\sigma^{*}$ shape-resonances observed in XANES. We demonstrate that these peaks result from coherent, high-order MS. We also obtain a generalization of the Natoli rule correlating the resonance locations with bond length.
\end{abstract}

Keywords: XAFS, XANES

\section{INTRODUCTION}

Curved-wave multiple-scattering (MS) theory') provides a unified theory for $x$ - $t$ ay-absorption fine structure (XAFS) that encompasses both the extended (EXAFS) and near-edge (NEXAFS) regimes. When carried to all orders, this theory is equivalent to exact treatments based on wave functions and Hamiltonian diagonalizations. In this talk we briefly review recent progress progress in curved-wave MS calculations and on understanding the nature of MS contributions in EXAFS and XANES. We focus particular attention on three computational difficulties in calculating MS to high order: i) the large angular momentum indices needed at high energies; ii) the proliferation of MS paths, and iii) MS DebyeWaller factors. We illustrate our findings with several examples where high order MS plays a significant role: the XAFS of $\mathrm{Cu}$ and Ti metals and the "shape resonances" in molecular $\mathrm{O}_{2}$.

\section{MULTIPLE SCATTERING EXPANSION}

The normalized XAFS is defined as $\chi=(\mu-$ $\left.\mu_{0}\right) / \mu_{0}$. where $\mu$ is the $x$-ray-absorption coefficient and $\mu_{0}$ the smooth atomic-like background. The absorption $\mu$ is proportional to the projected photoelectron density of states or. equivalently. to the imaginary part of a Green's-function matrix element. The result can be expressed as a over all MS paths $\Gamma_{1}^{1)}$ $\gamma=\Sigma_{\Gamma} \operatorname{Im}\left\langle e^{2 i \delta_{1}} \Sigma_{m}\left\langle 1 m\left|G t_{\because} \cdots G i_{2} G t_{1} G\right| 1 m\right)\right\rangle$ (in ma- trix notation). Here $G$ is a free-electron propagator, $t_{i}$ is the scattering $t$-matrix at site $i, \delta_{1}$ is the $l=1$ partialwave phase shift at the absorbing atom, and the outer brackets indicate a thermal and configurational average. We now briefly review some of the methods of calculation in common use.

\section{COMPUTATIONAL METHODS}

\subsection{Full-multiple scattering methods}

Full MS methods are equivalent to exact diagonalizations of the Hamiltonian and implicitly include MS tc all orders. One of these, the band structure method, is appropriate to crystalline solids and is based on calculations of the final state density of states. ${ }^{2)}$ It has been found to give the XAFS and XANES of metals in reasonable agreement with experiment in the low energy ( 0 $150 \mathrm{eV}$ ) range. However, the calculations usually ignore the core-hole and use ground-state potentials, leading to a systematic shift of the theoretical XAFS peaks with energy. The analog of the band-structure approach in molecules is the the $X_{\alpha}$ scattered wave method. ${ }^{3)}$ This approach has been extensively used for the XANES of molecules and small clusters. Good discussions of this method have been given by Kutzler et al, ${ }^{3)}$ and more recently by Tyson et $\mathrm{al}^{4}{ }^{4}$ ) the latter authors discuss MS more generally using both full MS and low-order MS methods. Drawbacks of these full MS approaches are the muffin-tin potential approximation, and the limitation to low-energies. Generalizations which allow for correc- 
tions to the muffin-tin approximation have been treated by Natoli et al. ${ }^{5)}$ An alternative method, the DVM (discrete variational method) of Ellis and co-workers ${ }^{6}$ ) uses a local basis which avoids the muffin-tin approximation, but is also limited to low energies.

\subsection{Cluster MS Methods}

An important advance in MS calculations was made by Durham et al. $\left.{ }^{7}\right)$ through the development of a cluster based MS method, which recognizes the importance of scattering in the neighborhood of the absorbing atom. This approach attempts to include the most important MS contributions by calculating MS to all orders within a given coordination shell but only a limited amount of intershell scatterings. The method has been improved by Vvedensky et al ${ }^{8)}$ to control the number of scatterings taken into account. The method has been used extensively for XANES calculations, but computational bottlenecks limit its use to low energies and small clusters.

\subsection{Path by Path MS}

\subsubsection{Exact Path Methods}

The path-by-path approach for calculating XAFS was pioneered largely by Lee and Pendry. ${ }^{1)}$ However, exact calculations are time-consuming and could only be carried out for single-scattering and a few low-order MS paths. By using the plane-wave-approximation (PWA), the method could be taken to high-order, but that approximation only works in the high-energy EXAFS regime with an inner-potential shift.

\subsubsection{Small Atom Approximations}

Many attempts to improve the PWA have been made, using variants of the small atom approximation (SAA). ${ }^{9)}$ These methods show that curved wave corrections are actually important throughout the XAFS regime, and introduce a phase shift of order $l(l+1) / k R$ into each partial wave. Methods based on the SAA have reasonable accuracy for EXAFS but lose accuracy at low energy, especially for non-collinear paths. In our view they are now supplanted by more accurate methods but do serve to illustrate the basic theory.

\subsubsection{Rapid-exact methods}

A major advance in MS calculations was made by Müller and Schaich, ${ }^{10}$ ) who showed using angular momentum identities that the exact curved-wave calculations could be calculated in closed form. This was extended by Gurman et al and others, ${ }^{11}$ to permit fast, exact calculations of low order (up to triple-scattering) MS paths. This degree of approximation gives accurate EXAFS calculations, but becomes time consuming at high energies and loses accuracy in the XANES region where high-order MS becomes important.

\subsubsection{Continued Fraction Method}

An alternative approach is the recently developed continued-fraction method to sum the MS series exactly for paths or clusters. ${ }^{12)}$ This method is potentially faster than the MS cluster methods, but becomes timeconsuming at high energies.

\section{SCATTERING MATRIX APPROACH}

Our own work is aimed at overcoming the major computational difficulties in current XAFS calculations: 1) the large angular momentum basis; 2) the proliferation of paths; and 3) the lack of MS Debye-Waller factors.

\subsection{Angular Momentum Bottleneck}

The angular momentum bottleneck was solved by using rotation matrices to rotate successive bonds in a path along the $z$-axis and then using an exact separable representation of the $z$-axis propagators. This permits a matrix factorization of the full propagator of the form $G_{L, L^{\prime}}=\left(e^{i k R} / k K^{\prime}\right) \Sigma_{\lambda} \Gamma_{L, \lambda} \bar{\Gamma}_{\lambda, L^{\prime}}$, which we will call the $\mathrm{RA}$ approach. ${ }^{13)}$ The use of $z$-axis propagators alone is a significant improvement in efficiency and they are also very accurate at very high energies but they do not remove the angular momentum bottleneck. The efficiency of the RA approach is due to this separable representation. It gives an accurate curved-wave XAFS formula analogous to that with the PWA, but with the usual scattering amplitudes $f(\theta)$ replaced by low-order (typically $6 \times 6$ ) matrices $F$. For an N-leg path $\Gamma$ with scatterers at $\vec{R}_{1}, \vec{R}_{2}, \ldots, \vec{R}_{N}=\vec{R}_{0}$, the result is

$$
\begin{aligned}
\chi \Gamma(p)= & \operatorname{Im} \quad S_{0}^{2} \frac{e^{i\left(\rho_{1}+\rho_{2}+\cdots \rho_{N}+2 \delta_{1}\right)}}{\rho_{1} \rho_{2} \cdots \rho_{N}} e^{-\sigma_{\Gamma}^{2} p^{2} / 2} \\
& \times \operatorname{Tr} M F^{N} \cdots F^{2} F^{1} .
\end{aligned}
$$

Here $\vec{\rho}_{i}=p\left(\vec{R}_{i}-\vec{R}_{i-1}\right), p=\sqrt{E-V_{m t}}$ is the photoelectron momentum measured with respect to the muffin-tin zero (in Rydberz-atomic units), $F^{i}$ is the scattering matrix at site $i, M$ is the $l=1$ termination matrix, $S_{0}^{2}$ is a many-body reduction factor, and $\sigma_{\Gamma}^{2}$ is the meansquare variation in total path length $R_{t}$. We have verified the adequacy of $6 \times 6$ matrices by using the $z$-axis propagators $\left.{ }^{13}\right)$ that are virtually exact, but slower by one or more orders of magnitude.

\subsection{Path Proliferation Bottleneck}

To overcome the path proliferation bottleneck, we restricted the number of paths being considered by introducing "filters" in the path enumeration scheme. It turns out that the majority of .MS paths are numerically insignificant, but it is not always obvious a priori which paths are most important. To automate this procedure, the contribution of a given MS path was estimated using the PWA, and only those paths of amplitude larger than a given cutoff were retained. This was done with 
a constructive heap algorithm. ${ }^{14)}$ To deal with degeneracies, physically equivalent paths are hash-sorted and only those with amplitudes above a second cutoff are retained. With these filters only a few percent of the MS paths need to be calculated to yield XAFS accurate to a few percent. Typically this amounts to fewer than 100 paths.

\subsection{Wultiple scattering Debye-Waller Factors}

One of the least satisfactory aspects of most MS treatments is the neglect of or crude treatment of vibrational and configurational disorder. However, it is relatively straightforward to include in a path by path treatment. Denoting the generalized coordinates of a given path by $x_{i}$, and assuming small or gaussian disorder, one obtains $<\chi_{\Gamma}>=\chi_{r}\left(<x_{i}>\right) W$, where $W=\exp \left[\partial^{2} \chi / \partial x_{i} \partial x_{j}\right)<x_{i} x_{j}>/ 2$ ! $]$ is the MS DebyeWaller factor. This kind of approach has been used by Benfatto et al. ${ }^{15}$ ) To simplify the task for practical calculations we have simply ignored angular variations and assumed only radial disorder in the XAFS phase. Thus $W=\exp \left(-\sigma_{\Gamma}^{2} k^{2} / 2 !\right)$, where $\sigma_{\Gamma}^{2}=$ $\Sigma_{i j}\left\langle\left(\vec{u}_{i}-\vec{u}_{i^{\prime}}\right) \cdot \dot{R}_{i i^{\prime}}\left(\vec{u}_{j}-\vec{u}_{j^{\prime}}\right) \cdot \dot{R}_{j^{\prime}}\right\rangle$, and $\vec{R}_{i j}=\vec{R}_{i}-\vec{R}_{j}$, $i^{\prime}=i+1, j^{\prime}=j+1$. For simplicity, we approximate the displacement-displacement correlation functions using an isotropic Debye model, ${ }^{16)}$ with a single parameter, the Debye-Temperature $\Theta_{D}$. An important consequence is that MS Debye-Waller factors contribute significantly to the convergence of the MS expansion, especially in the XANES region, since high-order MS paths are exponentially suppressed.

\section{EXAMPLES}

We now illustrate our approach with a few illustrative examples based on our automated XAFS code FEFF, 17-18) largely summarized from Ref. 18. Several others are summarized in these proceedings. ${ }^{19)}$

\section{$5.1 C u$}

Our first example is a discussion of MS in Cu metal. ${ }^{18)}$ Because of its close-packed structure and strong scattering potentials, fcc $\mathrm{Cu}$ poses a severe test for MS theories. Indeed, there are of order $10^{6}$ paths of length less than the typical mean-free path (about $20 \AA$ ), which determines the minimum cluster size needed for full MS calculations. We will concentrate this example on XAFS in position space, i.e., on the minimum number of MS paths needed to treat XAFS contributions out to a given maximum path length. This approach is useful in the analysis of XAFS experiments, where long-path MS can be eliminated by Fourier filtering. ${ }^{20}$ ) We used scattering phase shifts based on overlapped atom potentials which take the core-hole potential and the self-energy into account. as calculated using FEFF ${ }^{17-18}$ ). Our results for the $X A F S k^{2} \times\left(k^{2}\right), k=\sqrt{E-E_{F}}$, and for the Fourier

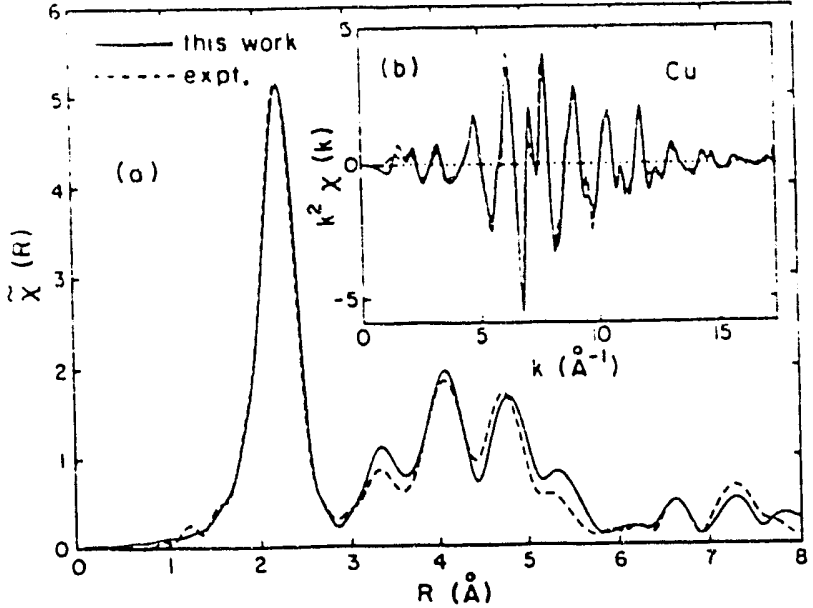

Fig. 1. Comparison of high-order MS calculations of $\mathrm{Cu} X A F S^{18)}$ with $S_{0}^{2}=0.906$ (solid line) and from XAFS experiment ${ }^{21)}$ at $190 \mathrm{~K}$ (dashed line): (a) position space Fourier transform $\dot{x}(R)$ and (b) $k^{2} x(k)$.

transform $\tilde{\chi}\left(R_{t} / 2\right)$ (Figure 1 ) are in reasonable agreement with experiment ${ }^{21)}$ with only one adjustable parameter, an overall amplitude factor $S_{0}^{2}=0.906$. Only 15 of the 32 total paths with $R_{t} / 2 \leq 5.1 \AA$ survived our cutoff filters set at $4 \%$ of the mean first-shell XAFS amplitude and suffice to describe $\bar{\chi}(R)$ out to the 4ih shell; none with more than triple scattering. Also, only 54 of the 17134 paths with $R_{t} / 2 \leq 8.466 \mathrm{~A}$, were needed out to the 8 th shell and, of those 54 , paths with 5 and 6 legs were found to be collinear. We have also demonstrated that the MS expansion converges to the results of full MS band-structure calculations ${ }^{22}$ ) using up to $10^{5}$ paths to of length less than $23.4 \AA$.

\section{$5.2 T i$}

Similar MS calculations were carried out for hcp Ti, in an effort to investigate the need for high-order MS in XANES. For this case we computed the background absorption $\mu_{0}$ using the dipole approximation and relativistic wavefunctions on an absolute energy scale, based on total energy calculations of the absorbing atom with and without the core-hole. In this example we also used the same $4 \%$ amplitude cutoff and a maximum distance of 6.58 Ato the 8 th shell, which gave a total of 8 singlescattering and $24 \mathrm{MS}$ paths. Finally, to compare with recent calculations of Vedrinskii et $\mathrm{al}^{23}$ ) we used two different self-energies. Our results (Figure 2) somewhat favor the hybrid self-energy with a shift of a few eV, but both are in iair agreement with experiment.

\section{$5.3 \quad \mathrm{O}_{2}$}

As a third example, we consider the diatomic molecule $\mathrm{O}_{2} .{ }^{18)}$ This calculations provides another severe test for IIS theories because of the short bond length and strong 


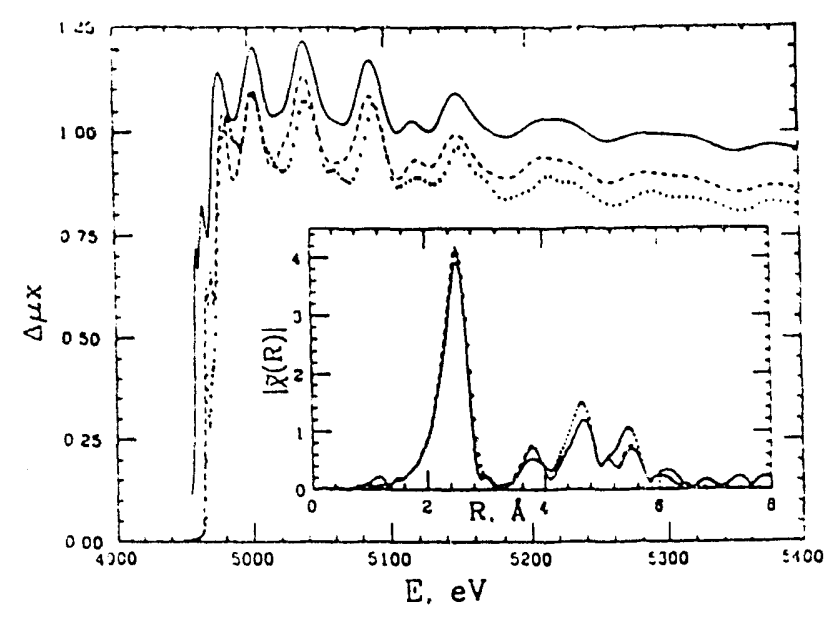

Fig. 2. MS calculations of the XANES of hcp Ti 4 vs absolute energy $E$ for two self-energy models: Hedin-Lundqvist-Quinn (dashed line), a hybrid model with a Dirac-Hara real part and Hedin-Lundqvist-Quinn imaginary part (solid line), and for comparison (squares) the experimental spectrum. ${ }^{24)}$ The inset is the Fourier transform $\dot{x}(R)$.

low-energy scattering in low- $Z$ atoms. A primary goal of this study was to understand the $\sigma^{*}$ shape resonances, ${ }^{25}$ ) which are of much interest since their locations in energy are correlated with bond length. ${ }^{26}$ ) The characteristic asymmetry of the peaks is similar to that of threedimensional square-well resonances ${ }^{27}$ ) but their fundamental origin has not been understood. Recently it was shown that the $\sigma^{*}$ resonances are well represented by $\mathrm{X} \alpha$-SW calcuiations and correspond to the first enhanced XAFS oscillation. ${ }^{28)}$ Subsequently we gave ${ }^{18)}$ an alternative XAFS interpretation based on high-order MS. In particular we found that the resonances in $\mathrm{O}_{2}$ and $\mathrm{N}_{2}$ can be explained by successive MS contributions from repeated bounces between the two scattering sites. Our calculations were carried out using an "extended-continuum" 4) modification of our codes. ${ }^{17-18)}$ Our results for $\mathrm{O}_{2}$ are illustrated in Figure 3.

Figure 3(a) shows how the resonance grows and sharpens as successive MS paths are added. The ove-all agreement with $\mathrm{X} \alpha-\mathrm{SW}$ calculations ${ }^{28}$ ) was surprising since our potential is not self-consistent and ignores the outersphere potential. We found that the large backscattering amplitude and the coherence of the MS phases control the resonance, and lead to a generalization of the Natoli rule relating peak position with bond length: ${ }^{26)}$ From Eq. (1) the MS phases vary as $(n+1) p R+2 \delta_{1}+\Phi_{n}$, where $R$ is the near-neighbor distance and $n$ is the number of backscatterings, so the condition for constructive interference of successive MS paths is $2 p R+2 \Delta \Phi=2 m \pi$ where $2 \Delta \Phi=\left(\Phi_{n+2}-\Phi_{n}\right)$ and $m$ is an integer, independent of $\delta_{1}$. Hence the peak location(s) satisfy

$$
p=(m \pi-\Delta \Phi) / R
$$

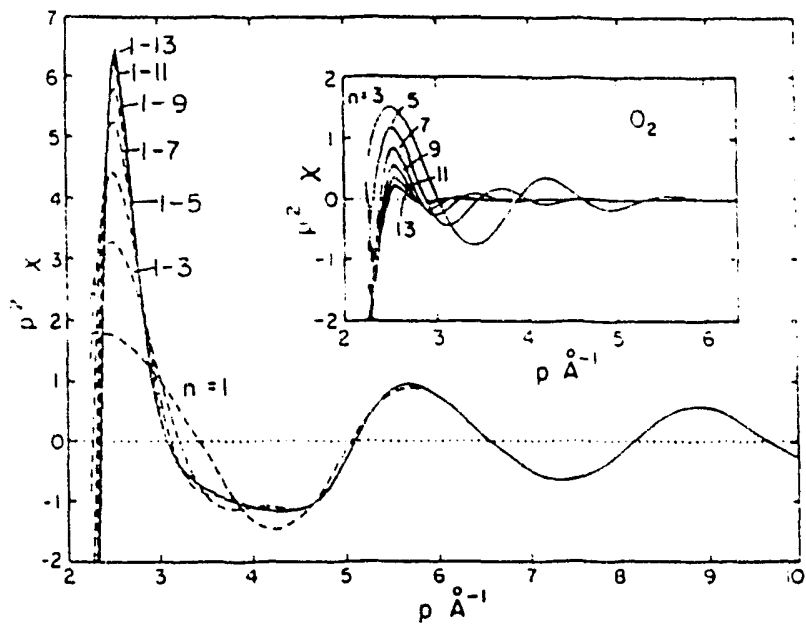

Fig. 3. MS calculations $\left.{ }^{18}\right)$ of the NEX AFS $p^{2} \times$ vs photoelectron momentum $p$ for molecular $\mathrm{O}_{2}$ : a) full spectrum with up to $n=13$ backscattering (solid lines) and partial sums with all paths up to a given $n$ (dashed lines); and b) individual MS contributions $p^{2} \times n$ with $n$ backscstterings.

In general the location of shape-resonance peaks may be expected to correspond to a stationary phase point (modulo $2 \pi$ ) of some subset of MS paths. For diatomic molecules, $\Delta \Phi$ is approximately twice the backscattering phase. Figure $3(\mathrm{~b})$ shows that the individual contributions $\chi_{n}$ from each MS path are restricted to successively lower wave-numbers. This is due in part to the MS Debye-Waller factors $\exp \left[-(n+1)^{2} \sigma^{2} p^{2} / 2\right]$, where for $\mathrm{O}_{2}, \sigma^{2}(300 K)=0.00136 A^{2}$, and shows how the Debye-Waller factors significantly improve the convergence of the MS series. This observation also suggests a distinction between NEXAFS and EXAFS on the basis of MS content. Similar calculations for $\mathrm{N}_{2}$ showed ${ }^{18}$ ) that the shape of the resonance is in good agreernent with experiment. ${ }^{27)}$

\section{CONCLUSIONS}

Although a number of methods have been developed for calculating MS, we have found that neither full-MS nor low-order-MS theories are fully satisfactory: loworder theories generally contain too little MS, full-MS theories generally contain too much, since many MS paths are of negligible importance, and are smeared out by inelastic losses and thermal disorder. Although high order MS calculations are desirable, they are difficult to compute using exact methods due to the sheer number of MS paths. Moreover approximate treatments, such as the PWA or SAA give uracceptable errors and the large angular momentum basis needed in exact treatments is a severe computational bottleneck. Another problem is the effect of thermal vibrations and disorder which have pronounced effects on the MS.

To overcome these bottlenecks. we have developed a 
high-order MS approach that permits a unified treatment of XAFS at both low and high energies. ${ }^{18,29)}$ Our pathby-path approach has a number of advantages over exact methods. In particular, the approach permits a geometrical interpretation of XAFS, handles arbitrarily high order MS in arbitrarily large systems, builds in inelastic losses and disorder, and is easier to compute than wavefunction methods. Our results support the observation that "shadowing"1) is an important element of the MS in EXAFS, but also show that it is not the only consideration; noncollinear MS accounts for a substantial fraction of the total amplitude. We also find that a relatively small number of MS paths suffice to represent the contributions in XAFS from the first few shells, in support of position-space XAFS analysis methods. ${ }^{20)}$ Our results confirm that noncollinear MS is important in the near-edge ${ }^{30}$ ) but they counter arguments that a full-MS treatment or full MS within a given shell is necessary. ${ }^{7-8)}$

ACKNOWLEDGMENTS: Our collaborators in this work are R.C. Albers and S.I. Zabinsky. We also thank K. Baberschke, J. Mustre de Leon, C.R. Natoli, J. Stohr, E. Stern for many useful discussions and M. Newville for assistance with the experimental comparisons. This work was supported in part by the U.S. Department of Energy (RCA) and by DOE grant DE-FG06-ER45415A003 (JJR).

\section{REFERENCES}

1) W. L. Schaich, Phys. Rev. B 8, 4028 (1973); P. A. Lee and J. B. Pendry, Phys. Rev. B 11, 2795 (1975); J. J. Barton and D. A. Shirley, Phys. Rev. B 32, 1906 (1985).

2) J. E. Müller, O. Jepsen, and J. W. Wilkins, Solid State Commun. 42, 365 (1982).

3) See for example, F. W. Kutzler, C. R. Natoli, D. K. Misemer, S. Doniach, and K. O. Hodgson, J. Chem. Phys.73, 3274 (1980), and references therein.

4) T. A. Tyson, K. Hodgson, C. R. Natoli, and M. Benfatto, Stanford Univ. Phys. Rev. B (to be published).

5) C. R. Natoli, M. Benfatto and S. Doniach, Phys. Rev. A34, 4682 (1986).

6) D. E. Ellis and G. L. Goodman, Int. J. Quantum Chem. XXV, 185 (1984).

7) P. J. Durham, J. B. Pendry and C. H. Hodges, Comp. Phys. Comm. 25, 193 (1982).

8) D. D. Vvedensky, D. K. Saldin, and J. B. Pendry, Comp. Phys. Comm. 40, 421 (1986).

9) See, for example. J. J. Rehr, R. C. Albers, C. R. Natoli. and E. A. Stern Phys. Rev. B 34, 4350 (1986), and references therein.

10) J. E. Müller and W. L. Schaich, Phys. Rev. B 27, 6489 (1983).

11) S. J. Gurman. N. Binsted, and I. Ross. J. Phys. C19.1845 (1986); M. F. Ruiz-Lopez, M. Loos, J. Goulon. M. Benfatto, and C. R. Natoli, Chem. Phys.
121, 419 (1988); R. V. Vedrinskii and L. A. Bugaev, J. Phys. B 24, 1967 (1991).

12) A. Filipponi, J. Phys. 3, 6489 (1991).

13) J. J. Rehr and R. C. Albers, Phys. Rev. B 41, 8139 (1990).

14) S. I. Zabinsky and M. J. Eller, unpublished.

15) M. Benfatto, C. R. Natoli and A. Filipponi, Phys. Rev. B40, 9636(1989).

16) E. Sevillano, H. Meuth, and J. J. Rehr, Phys. Rev. B 14, 9514 (1976).

17) J. J. Rehr, J. Mustre de Leon, S. I. Zabinsky, and R. C. Albers, J. Am. Chem. Soc 113, 5135 (1991); J. Mustre de Leon, J. J. Rehr, S. I. Zabinsky and R. C. Albers, Phys. Rev. B 44, 4146 (1991).

18) J. J. Rehr, S. I. Zabinsky, and R. C. Albers, UW Preprint (May, 1992).

19) See for example, P. Livins, J. J. Rehr, and R. Ingalls, (this proceedings); G. Pfeiffer, J. J. Rehr and D. A. Sayers (this proceedings).

20) J. Mustre, Y. Yacoby, E. A. Stern and J. J. Rehr, Phys. Rev. B 42, 10843 (1990).

21) E. A. Stern, B. A. Bunker and S. M. Heald, Phys. Rev. 21, 5521 (1980).

22) R. C. Albers, A. K. McMahan, and J. E. Müller, Phys. Rev. B 31, 3435 (1985).

23) L. Bugaev, R. V. Vedrinskii, I. G. Levin, and V. M. Airapetian. J. Phys. 3, 8967 (1991).

24) M. Newville (private communication).

25) See, for example, J. Stöhr, NEXAFS Spectroscopy (Springer, Heidelberg, 1992).

26) C. R. Natoli, EXAFS and Near Edge Structure, edited by A. Bianconi et al. (Springer, New York 1983), p. 43.

27) D. Arvanitis, H. Rabus, L. Wenzel, and K. Baberschke, Z. Phys. D 11, 219 (1989); Phys. Rev. B 40, 6409 (1989).

28) J. Stöhr and K. R. Bauchspeiss, Phys. Rev. Lett. 67, 3376 (1991).

29) Those interested in obtaining our MS codes FEFF ${ }^{(}$ should contact the authors.

30) G. Bunker and E. A. Stern, Phys. Rev. Lett. 52, 1940 (1984). 

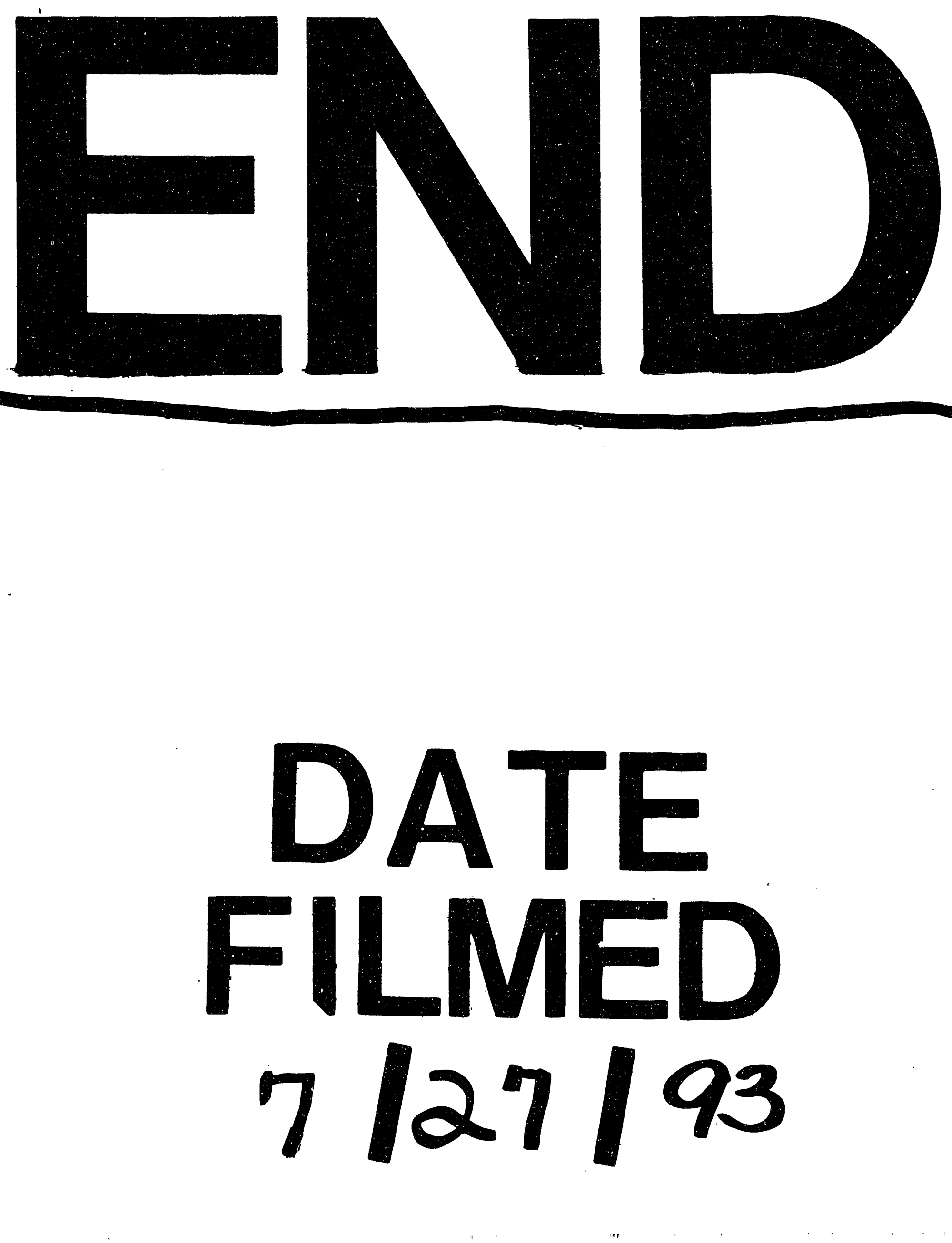
